\title{
Design and Implementation of Electronic Map of Agricultural Materials sales point Based on GIS
}

\author{
Huifang Bao ${ }^{1, a}$, Linli Zhou, ${ }^{1, b, *}$, Lei Liu ${ }^{2, c}$, Yue $\mathrm{Wu}^{2, \mathrm{~d}}$ \\ ${ }^{1}$ Institute of Intelligent Machines Chinese Academy of Sciences,Hefei 230031, China \\ ${ }^{2}$ University of Science and Technology of China,Hefei230026, China \\ a 1069484572@qq.com, ${ }^{\mathrm{b}}$ linlizhou@iim.ac.cn, ${ }^{\mathrm{c}}$ liulei12@mails.ucas.ac.cn, ${ }^{\mathrm{d}}$ wyue@iim.ac.cn
}

Keywords: maps API, agricultural materials, electronic map, retrieval, address resolution.

\begin{abstract}
In view of the shortcomings of high development cost and complicated maintain of traditional GIS platform, this paper put forward the design and implementation of electronic map system for agricultural material company on the basis of Baidu map API. Firstly, this paper summarized the features and basic functions of WebGIS, and proposed the service technical framework of electronic map based on the map API; Secondly, according to the needs analysis and feasibility analysis, determined the overall design block diagram of the system function module; Finally, the function design and implementation of each module were introduced in detail.
\end{abstract}

\section{Introduction}

With the development of computer network and application, people's demand for electronic information is becoming more and more strong, more and more rigorous, which makes a lot of scholars and experts to realize the huge development potential of cartography combined with computer science, the electronic map came into being ${ }^{[1]}$. The electronic map takes the visual digital map as the background, the amount of information that it represents is much larger than that of a traditional map. Compared with the traditional paper map, electronic map can conveniently combine and edit the contents of the general map in any form so as to form a new map. And the electronic map can be output at any scale or range. It can also be easily combined with other information sources such as satellite images, aerial photographs and so on to generate new maps ${ }^{[2]}$.

Agricultural materials refers to all kinds of material used in crop production to ensure the smooth progress of crop production, it includes chemical fertilizer, pesticide and seed, fertilizer, agricultural machinery etc.. Among them, chemical fertilizer, pesticide and seed are the most traditional and main inputs. The agricultural materials involved in this article are fertilizer, seed and pesticide.. China is a large agricultural country, but also a big consumer of agricultural materials. Therefore, the location, contact, telephone number and other info of agricultural materials company are very important information for consumers and enterprise managers. Through the acquisition of the above information, it can greatly facilitate consumers to purchase agricultural materials and access to value-added services. For managers of agricultural materials companies, they can intuitively understand the distribution of agricultural materials sales points of themselves and competitors, thereby strengthening management and making scientific decisions.

On the basis of Baidu map API, this paper has completed the design and implementation of electronic map of agricultural materials sales point. First of all, collect the basic information of the subordinate agricultural materials sales points of the company that has joined the system. And after the address translation, proofreading, typesetting and other processing to get the final data and upload it to the orca data management platform(Baidu map open platform to meet developers' various requirements for geographic data, open orca data management platform, so developers can maintain their geographic data in the platform). Then, mark the data on the map by calling the Baidu maps API, and realize the functions of online browsing, keyword retrieval, peripheral retrieval, rectangle area retrieval and address resolution and so on. Thus, which is convenient for consumers to get the fastest and best service, and is also beneficial for enterprise managers to plan as a whole. 


\section{WebGIS Introduction}

With the rapid development of Internet of things technology, location-based services based on Web GIS are getting more and more popular ${ }^{[3]}$. As a general network programming interface, map API has been widely applied in WebGIS ${ }^{[4]}$. Map API is an application program interface that embeds a map into a web page through JavaScript (or other programming language). Through this interface, developers can easily call information and functions in the database of map service websites, and build rich and interactive map applications. For developers, you only need to learn API development documents, and then you can complete almost all of the functions of the traditional GIS platform through integration and development. Moreover, the development cycle is short, the cost is low, the use is flexible, and it does not need to spend a lot of manpower and material resources to maintain the geographic information data ${ }^{[5]}$. According to the statistics, the number of sites calling map API has reached millions. At present, the mainstream map API services are Google Map API, Baidu map API, Tencent map API, Map-bar API and so on.The basic functions provided by the map API are as follows: basic map function, map retrieval, cover function, inverse / address resolution, control display function, event listeners, route planning, function of picture layer, positioning function and so on.

After investigation, we found that Baidu maps in the domestic access is very fast. Secondly, Baidu maps continue to open the API interface for free, and the number of calls to the interface is much higher than other electronic maps of peers. Therefore, the electronic map of the agricultural materials sales point in this system is developed on the basis of Baidu map API.

\section{Technical Framework of Electronic Map}

A complete electronic map basically contains three levels: client (browser), WEB server and database server ${ }^{[6]}$. The electronic map of the agricultural materials sales point based on map API discussed in this paper is also designed according to the above three layers B/S structure, as shown in figure 2.

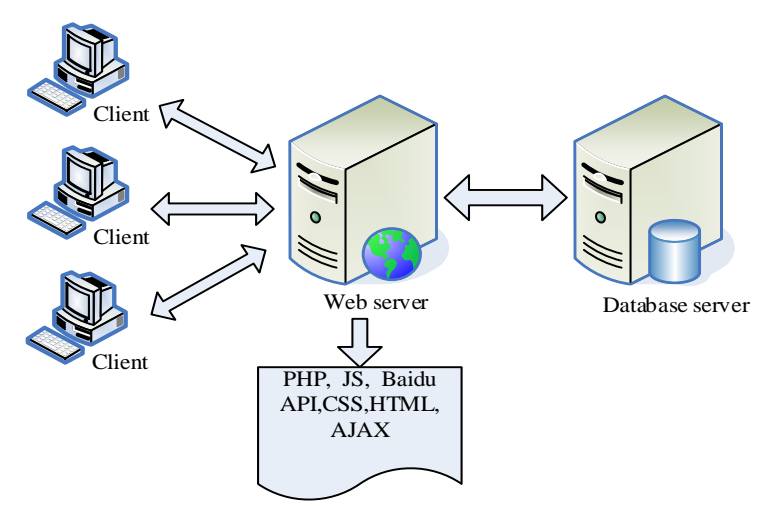

Fig.2 Technical framework of electronic map

(1) database server using orca data management platform for data storage, which mainly stores the latitude and longitude, address, name, service type, contact and other information of agricultural materials company .

(2) Web server adopts Apache server, and server-side uses PHP language as the scripting language. The front-end uses HTML+CSS+javascript+AJAX for design and development, CSS completes the style control, the JavaScript is responsible for calling the map API and interacting with the user, and the AJAX implements data update with page without refresh, wherein the data transmission process uses the XML format string in a unified way.

(3) on the client side, users can view the distribution of agricultural materials sales points visually through the browser, and do the following convenient operations: search, chart analysis, translation, scaling, etc... 


\section{Design and Implementation of Function Module}

\subsection{Block Diagram of Overall Structure.}

According to the requirement analysis and feasibility analysis, the main function modules and their relationship of this system is determined, this system is mainly composed of four functional modules: basic operation module, retrieval function module, statistical analysis based on Echarts, address resolution module.

\subsection{Basic Operation.}

The basic operations of the map include page composition, map browsing and info window.

(1) Page composition: The map page is composed of legend, navigation control, scale control, thumbnail control, map display area. the data information in the visible rectangle area is displayed in two ways: the map and the information list, as shown in figure 4 and figure 5 . Among them, red droplets represent integrated companies, blue droplets stand for fertilizer companies, yellow droplets stand for pesticide companies, and green droplets represent seed companies.

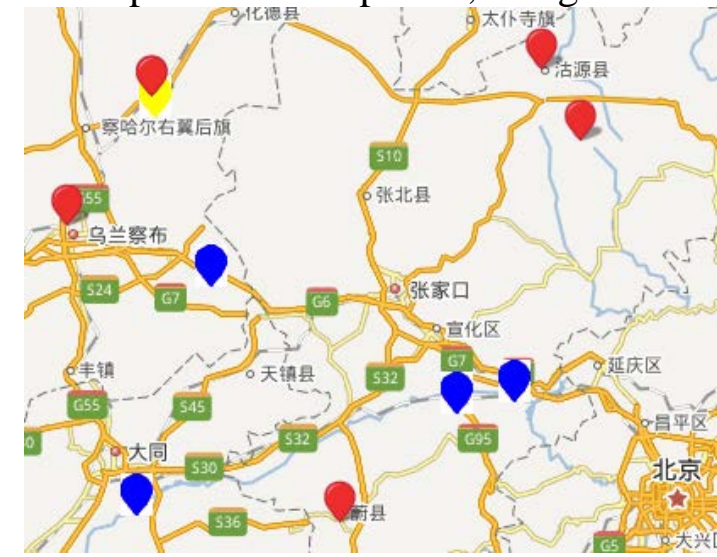

Fig.4 Data is displayed on map

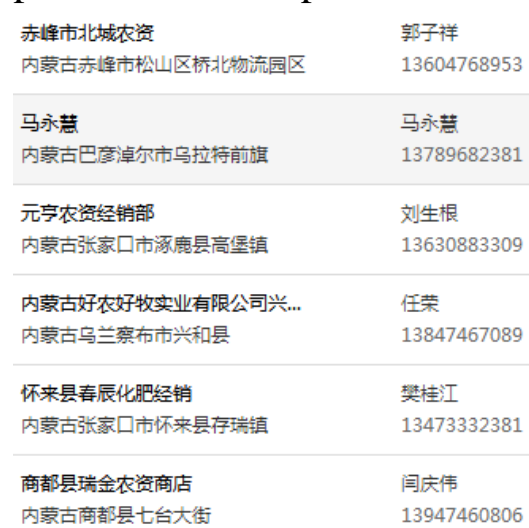

Fig.5 Data is displayed in a list

(2) Map browsing: mainly include zooming, draging, translation and other functions.

1) enlarging: double-click the right mouse button on any part of the map, or drag the zoom control to the "+ " direction, or slide the mouse wheel up, then the designated area of the map can be enlarged.

2) narrowing: double-click the left mouse button on any part of the map, or drag the zoom control to the "- " direction, or slide the mouse wheel down, then the designated area of the map can be narrowed.

3) translation: When the mouse is placed on the map, the cursor becomes a hand, then press and hold the left mouse button to translate map, or click the arrow on the navigation control to achieve the translation of the map.

(3)info window: Click on the small water droplets on the map will pop up the information window, display the details of the sales point, consumers can contact the sales point based on this information.

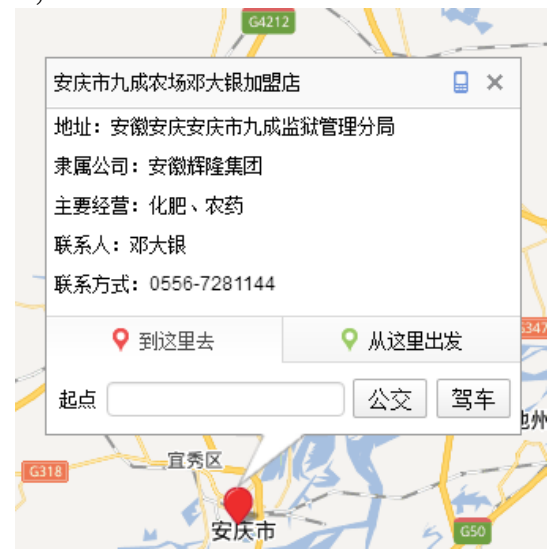

Fig.5 Info window 


\subsection{Address resolution module.}

The basic information of agricultural materials sales points collected from agricultural materials company usually contain only the address, type of service, service scope, contact person, telephone number and so on, but there is no latitude and longitude, which requires that the address information be resolved first to obtain its latitude and longitude coordinates before it can be marked on the map. Specific procedures are as follows: Firstly, we add this address information to the Excel table 1 . Then, by calling the address resolution interface of the Baidu map, the latitude and longitude of the address are obtained, And finally, the results are exported to the Excel table 2. In the parsing process, an exception handling mechanism is added to mark the addresses that cannot be parsed correctly. Later, ask the contact for the correct address information of the agricultural materials company. Address resolution is shown in Figure 8 below.

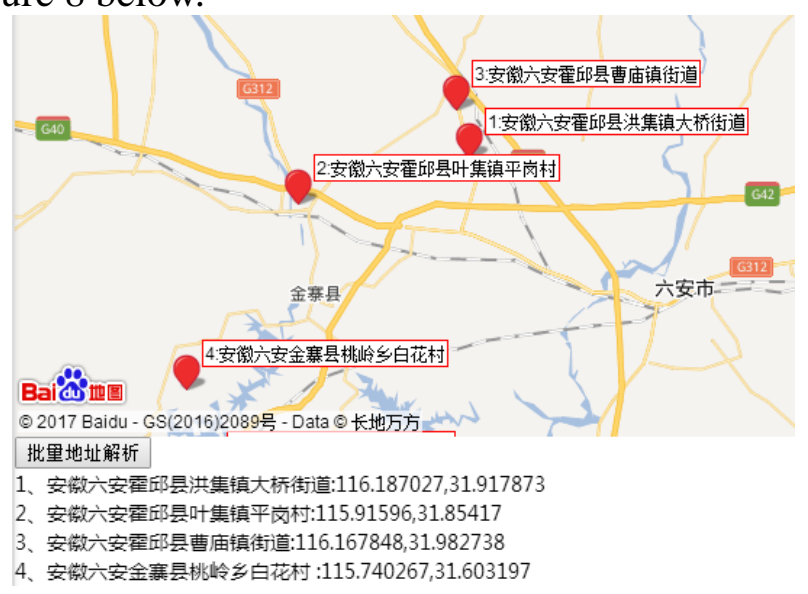

Fig.8 Address resolution display

\subsection{Statistical Analysis Based on Echarts}

This module carries on the statistical analysis to the massive data through the statistical chart, so that visitors are absolutely clear. Echarts is a very elegant and easy to use visual product launched by Baidu. It is a chart library based on Canvas and pure javascript, and provides visual, vivid, interactive and customizable visual charts. In this module, the pie chart in echarts is used for the statistical analysis of data, and the pie graph can be generated online according to the province information chosen by the user. When the province information changes, the pie chart structure changes accordingly. The principle is: first, use AJAX technology to call the data retrieval interface of Baidu map to obtain data, and then use js to call Echart function to realize the pie chart drawing. As shown in Figure 7, it shows the distribution of seed companies in selected provinces through pie chart.

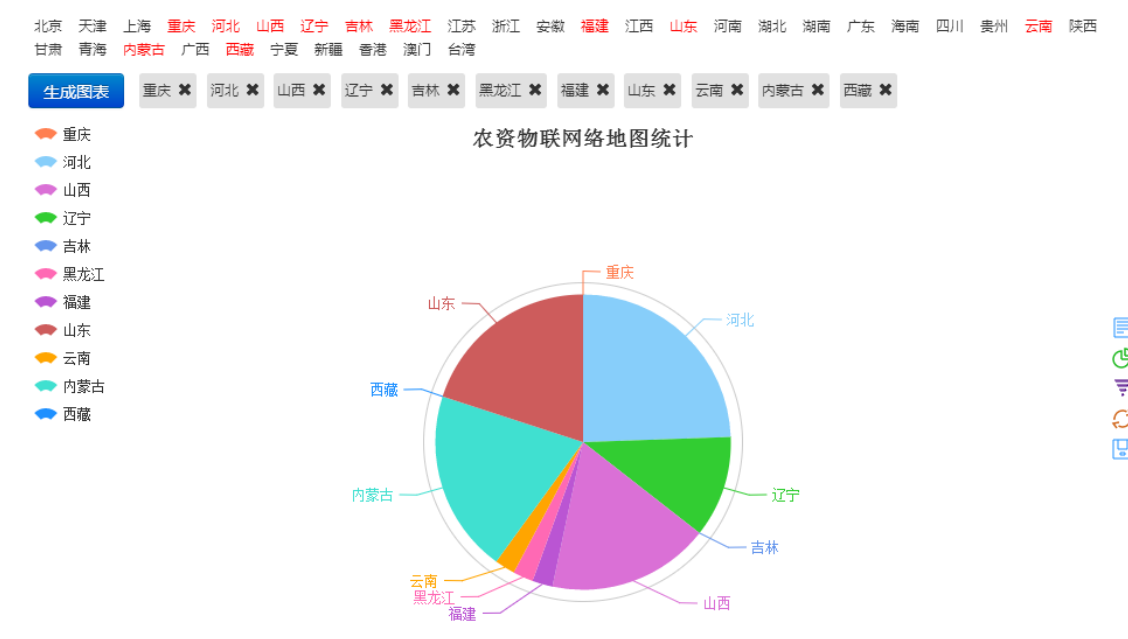

Fig.7 The statistical information of the pie chart

\subsection{Retrieval Function Module.}


Data retrieval is mainly divided into the following three cases:

Case 1: Rectangular area retrieval. When a drag event occurs or a zoom event caused by the mouse wheel occurs, the relevant data within the visible rectangular region is retrieved and were marked on the map;

Case 2: Keyword retrieval. When we build table, the four fields of name, address, contact, and management type are added to the retrieval attribute. when the keywords in the search box is changed, the contents of these four fields will be fuzzy matching. And the retrieval results were displayed by two ways: map and information list.

\section{Conclusions}

In view of the electronic map of agricultural materials sales point designed in this paper, the customer is the most direct beneficiary, they can easily find the nearest agricultural materials sales points from the electronic map, get more considerate service. And for managers of agricultural materials companies, they can intuitively understand the distribution of agricultural materials sales points of themselves and competitors, thereby strengthening management and making scientific decisions. Practice has proved that the use of map API to develop electronic map service system has the advantages of openness, low cost, low technical threshold, easy customization and so on. Which has broad application prospects.

\section{Acknowledgment}

This work was supported by the National Science \& Technology Pillar Program during the 12th Five-year Plan Period (Grant No. 2015BAD18B05)

\section{References}

[1]Qing Hui Wang,Wen Zhou Wang,Dan Li,Lei Chang. The Application of Electronic Map in Wireless Positioning System[J]. Advanced Materials Research,2013,2534(756).

[2]Lei Peng. Key Technology in a Lightweight WPF-Based Electronic Map Engine[J]. Applied Mechanics and Materials,2014,3561(678).

[3]Kilibarda Milan,Protić Dragutin,Nestorov Ivan. Application of Google Maps API service for creating web map of information retrieved from CORINE land cover databases[J]. Glasnik Srpskog Geografskog Društva,2010,90(4).

[4]Cong $\mathrm{Fu}$, Yan Wang, Yanqing $\mathrm{Xu}$, et al. The logistics network system based on the Google Maps API. in: Proceedings of 2010 International Conference on Logistics Systems and Intelligent Management. Harbin, China: IEEE Computer Society Press, 2010. 1486 1489.

[5]Gold, Jon. FourSquare drops Google Maps API for OpenStreetMap[J]. EN,2012.

[6]Lin Liu,Wan Wu Li. Related Technology Research on Navigation Electronic Map[J]. Applied Mechanics and Materials,2012,2077(236). 